\title{
The lived experience of children and adolescents with cancer
}

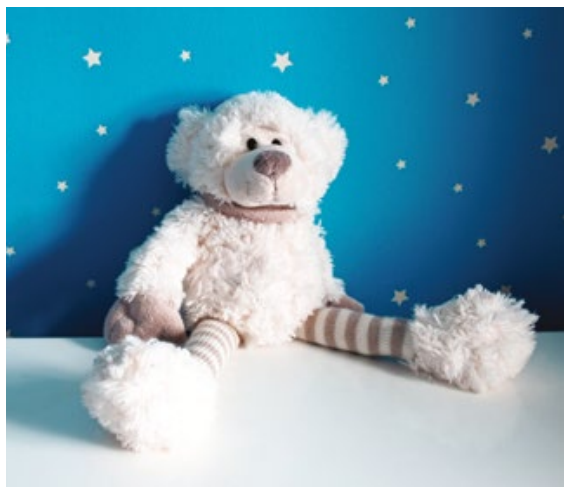

CPD

Jordana McLoone, Claire E Wakefield, Ursula M Sansom-Daly, Elysia Thornton-Benko, Dinisha Govender, Melissa Gabriel, Thomas Walwyn, Christina Signorelli, Karen Johnston, Richard J Cohn

\section{Background}

The lived experience of children and adolescents diagnosed with cancer differs greatly from that of the adult cancer patient. A diagnosis of cancer disrupts almost every developmental life stage and continues to affect the child, and potentially their whole family, throughout adulthood.

\section{Objective}

While it is important to recognise the potential for posttraumatic growth, a considerable proportion of children and adolescents will experience poorer psychological, social, educational and quality-of-life outcomes. Parents, particularly mothers, have been shown to experience levels of post-traumatic distress even greater than that of survivors. As such, there exists a critical need to provide family-centred support from diagnosis through to long-term survivorship or bereavement.

\section{Discussion}

Ongoing surveillance, proactive management of chronic health conditions, and health behaviour education are critical to survivors' lifelong wellbeing and can be facilitated locally by general practitioners with support from tertiary healthcare teams in a shared-care arrangement.

A CANCER DIAGNOSIS is rarely expected, but when it occurs in a child or adolescent, it is particularly shocking because of its relative rarity. Childhood cancer represents $<1 \%$ of all invasive cancers diagnosed in Australia, equating to roughly 770 new childhood cancer diagnoses per year. ${ }^{1,2}$ However, as the leading cause of disease-related death in children, a cancer diagnosis can be extremely distressing to the child or adolescent and their family. While families face high levels of uncertainty and the burden of treatment, the majority of children will survive, with five-year survival rates now reaching $80 \%$ in Australia. ${ }^{2}$ Such improvements in survival mean the survivorship period now extends across almost six decades. ${ }^{3}$ For adult survivors of child and adolescent cancer who were treated in earlier decades, up to $96 \%$ will develop an average of five severe, disabling or life-threatening chronic health conditions as late effects of their cancer or its treatment. ${ }^{4}$ Survivors who were treated in more recent times may experience far fewer treatment-related complications and potentially can be risk stratified to receive primary care follow-up. Regardless, the cancer journey for children, adolescents and their families is long and complex. The aim of this article is to describe the lived experience of children and adolescents with cancer, from diagnosis through to survivorship or bereavement, focusing on the implications for general practitioners (GPs). A brief summary is outlined in Table 1.

\section{Diagnosis and treatment}

Young people can experience a range of emotional responses when they initially receive a cancer diagnosis, including shock, grief, anxiety and anger. ${ }^{5}$ Some young people report feeling a sense of 'numbness' or 'surrealism'; this can lead to an apparent absence of emotional reaction that can appear similar to avoidance or denial. While some young people do report actively trying to hide their emotions to shield or protect their families (as do many families/parents in reverse), for other young people an initial sense of denial can be protective, enabling them 
to absorb information in a slower, gradual manner as their capacity to process it develops. ${ }^{6}$ The start of cancer treatment often triggers an increase in distress, as their new cancer becomes more of a reality. The months' worth of missed school, social events and sports can come into clearer focus at this time and must be faced in tandem with the heavy burden of treatment protocols and side effects.

A cancer diagnosis in a young person fundamentally disrupts critical developmental stages. A key focus of gold-standard psychosocial care for children and adolescents with cancer is therefore to mitigate this developmental impact to the greatest extent possible. ${ }^{7}$ For younger children, missed schooling and opportunities for social development exert the greatest psychosocial impact during treatment. Among adolescents, for whom it is developmentally normal to be aligning more closely with their peer group, this loss of social connection can be particularly distressing ${ }^{5}$ and may be experienced in addition to body image concerns (eg hair loss). ${ }^{8}$ This can lead to social withdrawal, avoidance and related distress throughout the duration of treatment. The cumulative effects of missed school and treatment side effects can also affect school achievement, and an individual child's performance should be considered relative to their pre-cancer performance, not the class average. Recognising the potential mental health impact that any changes to learning ability and academic identity may have is key to supporting the child at school.

\section{Implications for general practitioners \\ GPs bring particular strengths to a} multidisciplinary team, most notably their knowledge of the whole-family dynamic and their capacity to provide holistic care, psychological support and referral. ${ }^{9}$ Psychological stress is often experienced at the completion of therapy and during the subsequent disease surveillance period, prior to the longer-term survivorship period. GPs will often have more involvement in this period of care than during therapy and can help the patients navigate this transition from patient to well person. Parents' experiences of cancerrelated financial toxicity should also be discussed as part of standard care, and referral made to professional services and community organisations that are able to provide assistance. ${ }^{10}$

\section{Survivorship}

The completion of cancer treatment is often an eagerly anticipated milestone that is celebrated by the child or adolescent, their family and their community. However, families must now traverse a path outside the cancer ward and can face more uncertainty and potentially less support. Survivorship research has become increasingly more sophisticated, with large cohort trials across Europe and the USA providing evidence that chemotherapy, radiotherapy and surgery result in clinically significant long-term physical, intellectual, emotional, social and occupational difficulties, with no plateau for the increased risk of adverse health conditions. ${ }^{11}$

For this reason, it is critical for children and adolescents to receive life-long cancer-related care, which may facilitate the early detection of late effects and the timely initiation of interventions to preserve and improve health. ${ }^{12}$ However, keeping informed of developments in survivorship care is a genuine challenge, with the number of survivorship studies quadrupling since $1996 .{ }^{13}$ Evidence-based clinical practice guidelines and tailored care plans to support risk stratification and shared care are needed, as non-oncology health professionals cannot be expected to maintain pace with this rapid expansion of the field. Childhood cancer-specific survivorship guidelines are available

\section{Table 1. Summary of experiences and the general practitioner's role, by cancer stage}

\begin{tabular}{lll}
\hline Stage & Lived experience & Role of the general practitioner \\
\hline Diagnosis & Shock, relief, fear, determination to overcome. & $\begin{array}{l}\text { Provide support, liaise with specialists, consider } \\
\text { impacts on other health conditions, consider impacts } \\
\text { on other members of the family. }\end{array}$ \\
& & on isolation, body image concerns.
\end{tabular}

Treatment Physical challenges: pain, fatigue, nausea. Emotional challenges: grief, anger, fear, sadness to lose normal life and activities. Coping with altered friendships, challenges to body image, fear of the future.

May experience an increased sense of routine or control. May need to make practical adjustments to accommodate severe/chronic illness.

Survivorship Uncertainty, fear of cancer recurrence, post-traumatic stress, post-traumatic growth. Financial toxicity. Fatigue, pain, multiple comorbidities.

Bereavement Grief, finding meaning, changes to family dynamic, changes to parent-child (sibling) and marital dyads.
Provide timely and regular communication with the patient and family to aid confidence and engagement. Recognise the potential impact of treatment on siblings and focus on meeting their needs.

Take a more active role in surveillance for long-term effects and specialist referral. Screen for possible cognitive impact of treatment. Promote positive health behaviours. Provide psychosocial support to the family.

It is very meaningful for families to talk to someone who knew their child and was involved in their care. The family's mental health is now foremost. 
online (www.survivorshipguidelines.org). Interventions are also needed to engage, educate and empower survivors to manage their own health (eg the Re-engage program, recently renamed Engage) ${ }^{14}$ and meet survivors' unmet information needs related to late effects, as well as parents' unmet information needs, particularly related to their child's fertility. ${ }^{15}$

Improving survivors' adherence to health behaviour guidelines is critical, as there now exists strong evidence that positive health behaviours are fundamental, effective prevention strategies to lower the prevalence of late effects of cancer. ${ }^{16}$ For example, maintaining higher levels of exercise over an eight-year period has been found to result in a $40 \%$ reduction of all-cause mortality among childhood cancer survivors, compared with the maintenance of lower levels of exercise. ${ }^{17}$ Unfortunately, most survivors do not comply with recommended health behaviour guidelines, including engaging in survivorship care (up to $68 \%$ of survivors are disengaged), ${ }^{18}$ physical activity (up to $52 \%$ of survivors are sedentary), ${ }^{19,20}$ fruit and vegetable intake (up to $93 \%$ of survivors are non-compliant), ${ }^{21,22}$ limiting sun exposure at peak ultraviolet radiation times, ${ }^{23}$ and abstaining from smoking (up to $22 \%$ of survivors take up smoking) ${ }^{23}$ and using e-cigarettes (vaping). ${ }^{24}$

GPs can play a critical part in promoting positive health behaviours in childhood cancer survivors. It is important to remember that not all survivors will experience a renewed sense of carpe diem after surviving cancer, nor be motivated to observe all health behaviour guidelines. For some adult survivors of childhood cancer, it is sometimes a daily battle to manage multiple comorbidities, pain, fatigue, fear of cancer recurrence and mental health concerns. ${ }^{25}$ The following case study illustrates the lifelong psychological and physical challenges that survivors often experience.

\section{CASE STUDY: A SURVIVOR'S VOICE}

$M y$ cancer surgery and treatment was all in my infancy. I don't remember it. However the surgical changes to my body and side effects of the chemotherapy have heavily affected my life, most notably, the orchidectomy, androgen deficiency, infertility, osteopenia, and post-traumatic stress disorder. These issues have always been such a difficult subject to discuss, even within my family. As an adolescent, I stopped attending follow-up care, as I had been off treatment for a decade, I didn't live in Sydney and there were a lot of other things going on.

In my teens I was concerned about my development in relation to my peers. My GP told me I was just a late bloomer. However, as a childhood cancer survivor, with a history of chemotherapy and a damaged testicle, my concerns were warranted. Intervention could have changed my life significantly. At age 20, I had a fertility test. My GP didn't think this was necessary, as I wasn't planning a family, but allowed it for peace of mind. It came back negative, azoospermia. We had a brief discussion and off I went. Of course, the GP couldn't change anything, but it would have been a great time to point me towards some help or support. I should have done it myself, but I was traumatised and I didn't deal with my infertility for over 10 years. Relationships rose and fell because of it.

My next dilemma was a testosterone deficiency. Over the years I had tried to discuss it with several GPs, they always said there was no need to check my testosterone, it will be fine. Embarrassed and humiliated because of my problems, I gave up until my new GP realised it was worth checking and that I had a testosterone deficiency. I was referred to a specialist who treated me like everyone else, ignored my history and put me through several years of torture explaining that my testosterone would pick up soon because we were following the recommended guidelines. I felt weak, humiliated and suicidal. Finally, when I re-engaged with the multidisciplinary team at the childhood cancer long-term follow-up clinic, I met a new endocrinologist who changed my life completely-instantly. She knew I had special requirements, which are not common to all, but are common to long-term childhood cancer survivors. The clinic staff have communicated with my GP to facilitate and guide areas in which I needed help, drawing on their expertise in survivorship issues.

I think mental health is something that is a major issue for survivors also. The GP
Medicare mental health plans have helped. I think for a man, it's easier to accept help for your mental health ifyour doctor is involved. It is all too hard to admit it to yourself and all too easy to give up after encountering difficulties. The anguish that hormone and reproductive health gave me over the years was phenomenal, and the feelings of defeat and humiliation I experienced while trying to get to the bottom of it was crushing and made me hesitant to try again.

I believe all the doctors I've seen have meant well and were trying their best to help me. However, I believe a bit more understanding and a little less reluctance to reach out for more information could really improve quality of life for long-term childhood cancer survivors like me. I am so grateful that I found a GP who would work with me, as I would be dead if I hadn't.

\section{Implications for general practitioners} GPs are central to promoting sustainable and lifelong health behaviours, which have the potential to reduce risk and improve survivor quality of life yet are not currently adopted by most survivors. Survivors need the support of GPs to manage ongoing follow-up care, oversee surveillance and provide specialist referral across the course of the decades' long survivorship period. ${ }^{26,27}$ Formal emotional support is rarely accessed by childhood cancer survivors and their families, ${ }^{28}$ and this can lead to survivors 'suffering in silence'. GP-initiated discussion of mental health and the formation of a mental health plan, as indicated, with ongoing regular psychological support, is recommended.

\section{Bereavement}

Even when it is not possible to save the child, it remains important to ensure the survival of the family. Bereaved parents report an intolerable pain, which continues to affect them throughout their lives. Bereaved parents are at increased risk of long-term mental health issues, reduced marriage quality, reduced social function, and occupational and financial disadvantage. ${ }^{29}$ Bereaved siblings often struggle to adjust to the loss of their 
brother or sister and the broader changes in parenting and family dynamics after the death of their sibling. ${ }^{29}$

\section{Implications for general practitioners}

Staying connected to their deceased child's healthcare professionals can prevent feelings of loss and abandonment for parents. GPs can help to ensure families continue to feel cared for, reducing their sense of isolation and increasing their sense of support. It is particularly important for the GP to provide such care, as hospital bereavement support can be limited, and families can have reduced contact with the hospital after their child ceases to receive treatment. Australian resources are available to help support health professionals have these difficult conversations (www.redkite.org.au/ resource/walking-alongside).

\section{Summary}

Collaborative care and management between oncologists, GPs, patients and families is key in working towards the shared goal of optimising quality of life for child and adolescent cancer survivors. The provision of ongoing medical care and surveillance for the management of multiple comorbidities and risks, as well as the provision of psychosocial support, is fundamental to ensure that children and adolescents diagnosed with cancer experience a life well lived. Excellent online cancer survivorship education resources for primary care professionals are now available (eg Future Learn offers 'Cancer Survivorship for Primary Care Practitioners' [www.futurelearn.com/ courses/cancer-survivorship], while eviQ Education offers introductory courses in paediatric cancer and survivorship care [https://education.eviq.org.au/courses/ supportive-care/cancer-survivorshipintroductory-course]).

\section{Key points}

- A diagnosis of cancer in a child or adolescent is a traumatic event for both the patient and their family.

- Although over $80 \%$ of children will survive, the journey to recovery is long and requires ongoing support from the point of diagnosis through to survivorship or bereavement.

- The majority of survivors of child and adolescent cancer develop long-term late effects from their cancer and its treatment. This risk increases with the passage of time. Late effects include morbidities related to the cancer diagnosis and treatment received, including multiple chronic health conditions, fatigue, pain and poor psychosocial adjustment.

- Ongoing surveillance for late effects and support to comply with recommended health behaviours is essential for maintaining health and wellness.

\section{Authors}

Jordana McLoone PhD, Lecturer, School of Women's and Children's Health, University of New South Wales, Sydney, NSW; Behavioural Sciences Unit, Kids Cancer Centre, Sydney Children's Hospital, Randwick, NSW

Claire E Wakefield PhD, Professor, School of Women's and Children's Health, University of New South Wales, Sydney, NSW; Behavioural Sciences Unit, Kids Cance Centre, Sydney Children's Hospital, Randwick, NSW Ursula M Sansom-Daly BPsych (Hons), MPsychol (Clin), PhD, Senior Research Fellow, School of Women's and Children's Health, UNSW Medicine, University of New South Wales, Randwick, NSW; Behavioural Sciences Unit, Kids Cancer Centre, Sydney Children's Hospital, Sydney, NSW; Sydney Youth Cancer Service, Nelune Comprehensive Cancer Centre, Prince of Wales Hospital, Randwick, NSW Elysia Thornton-Benko BSc, MBBS (Hons), PhD, FRACGP, Specialist General Practitioner, Sydney, NSW; Research Fellow, Behavioural Sciences Unit, Faculty of Medicine, University of New South Wales, Sydney, NSW; Kids Cancer Centre, Sydney Children's Hospital, Randwick, NSW

Dinisha Govender MBChB, FRACP, Staff Specialist, Children's Hospital at Westmead, Westmead, NSW Melissa Gabriel MBBS, FRACP, Head, Oncology Survivorship Clinic, Caner Centre for Children, Children's Hospital at Westmead, Westmead, NSW Thomas Walwyn MBBS, DRCOG, MRCPCH, FRACP, Paediatric \& Adolescent Oncologist, Perth Children's Hospital, Nedlands, WA

Christina Signorelli PhD, Post-doctoral Fellow" School of Women's and Children's Health, University of New South Wales, Sydney, NSW; Behavioural Sciences Unit, Kids Cancer Centre, Sydney Children's Hospital, Randwick, NSW

Karen Johnston, RN, Clinical Nurse Consultant, Kids Cancer Centre, Sydney Children's Hospital, Randwick, NSW

Richard J Cohn MBBCh, DCH, FRACP, Paediatric Haematologist Oncologist, Director of Survivorship Program, Kids Cancer Centre, Sydney Children's Hospital, Randwick, NSW; Professor, School of Women's and Children's Health, University of New South Wales, Sydney, NSW Competing interests: None.

Funding: The Behavioural Sciences Unit (BSU) is proudly supported by the Kids with Cancer
Foundation. JM is supported by the Medical Research Future Fund, Australian Brain Cancer Mission, Brain Cancer Survivorship Grant (MRFBC000002). CEW is supported by a Career Development Fellowship from the National Health and Medical Research Council of Australia (APP1143767). CS is supported by a CINSW Early Career Fellowship (2020/ECF1144). This work has been supported by a Medical Research Future Fund (MRFF) Brain Cancer Survivorship grant (MRFBC000002).

Provenance and peer review: Commissioned, externally peer reviewed.

Correspondence to:

j.mcloone@unsw.edu.au

\section{References}

1. Youlden DR, Baade PD, Green AC, Valery PC, Moore AS, Aitken JF. The incidence of childhood cancer in Australia, 1983-2015, and projections to 2035. Med J Aust 2020;212(3):113-20. doi: $10.5694 / \mathrm{mja} 2.50456$.

2. Baade PD, Youlden DR, Valery PC, et al. Population-based survival estimates for childhood cancer in Australia during the period 1997-2006. Br J Cancer 2010;103(11):1663-70. doi: 10.1038/ sj.bjc.6605985.

3. Yeh JM, Ward ZJ, Chaudhry A, et al. Life expectancy of adult survivors of childhood cancer over 3 decades. JAMA Oncol 2020;6(3):350-57. doi: 10.1001/jamaoncol.2019.5582.

4. Bhakta N, Liu Q, Ness KK, et al. The cumulative burden of surviving childhood cancer: An initial report from the St Jude Lifetime Cohort Study (SJLIFE). Lancet 2017;390(10112):2569-82. doi: 10.1016/S0140-6736(17)31610-0.

5. Sansom-Daly UM, Wakefield CE. Distress and adjustment among adolescents and young adults with cancer: An empirical and conceptual review. Transl Pediatr 2013;2(4):167-97. doi: 10.3978/j. issn.2224-4336.2013.10.06.

6. Lindsay T. Cancer, sex, drugs and death: A clinician guide to the psychological management of young people with cancer. Samford Valley, Qld: Australian Academic Press, 2017; p. 150.

7. Wiener L, Sansom-Daly UM. Interventions for children under age 15 living with cancer. In: Christ G, Messner C, Behar L, editors. Handbook of oncology social work: Psychosocial care for people with cancer. New York, NY: Oxford University Press, 2015; p. 447-55.

8. Brierley ME, Sansom-Daly UM, Baenziger J, McGill B, Wakefield CE. Impact of physical appearance changes reported by adolescent and young adult cancer survivors: A qualitative analysis. Eur J Cancer Care (Engl) 2019;28(4):e13052. doi: 10.1111/ecc.13052.

9. Noteboom EA, Vervoort SCJM, May AM, et al. Treatment decision-making and the added value of the general practitioner: A qualitative exploration of cancer patients' perspectives. Eur J Cancer Care (Engl) 2021;30(3):e13410. doi: 10.1111/ ecc.13410.

10. Kelada L, Wakefield CE, Vetsch J, et al. Financial toxicity of childhood cancer and changes to parents' employment after treatment completion. Pediatr Blood Cancer 2020;67(7):e28345. doi: $10.1002 /$ pbc. 28345 .

11. Norsker FN, Pedersen C, Armstrong GT, et al. Late effects in childhood cancer survivors: Early studies, survivor cohorts, and significant contributions to the field of late effects. Pediatr Clin North Am 2020;67(6):1033-49. doi: 10.1016/j. pcl.2020.07.002

12. Mulder RL, van Kalsbeek RJ, Hudson MM, Skinner R, Kremer LCM. The critical role of clinical practice guidelines and indicators in high-quality 
survivorship after childhood cancer. Pediatr Clin North Am 2020;67(6):1069-81. doi: 10.1016/j. pcl.2020.07.003.

13. Harrop JP, Dean JA, Paskett ED. Cancer survivorship research: $A$ review of the literature and summary of current $\mathrm{NCl}$-designated cance center projects. Cancer Epidemiol Biomarkers Prev 2011;20(10):2042-47. doi: 10.1158/1055-9965. EPI-11-0673.

14. Signorelli $C$, Wakefield $C E$, Johnston $K A$, et al. Re-engage: A novel nurse-led program for survivors of childhood cancer who are disengaged from cancer-related care. J Natl Compr Canc Netw 2020;18(8):1067-74. doi: 10.6004/ jnccn.2020.7552.

15. Vetsch J, Fardell JE, Wakefield CE, et al. "Forewarned and forearmed": Long-term childhood cancer survivors' and parents' information needs and implications for survivorship models of care. Patient Educ Couns 2017;100(2):355-63. doi: 10.1016/j. pec.2016.09.013.

16. McCullough ML, Patel AV, Kushi LH, et al. Following cancer prevention guidelines reduces risk of cancer, cardiovascular disease, and allcause mortality. Cancer Epidemiol Biomarkers Prev 2011;20(6):1089-97. doi: 10.1158/1055-9965. EPI-10-1173.

17. Scott JM, Li N, Liu Q, et al. Association of exercise with mortality in adult survivors of childhood cancer. JAMA Oncol 2018;4(10):1352-58. doi: 10.1001/jamaoncol.2018.2254.

18. Signorelli C, Wakefield CE, Fardell JE, et al. The impact of long-term follow-up care for childhood cancer survivors: A systematic review. Crit Rev Oncol Hematol 2017;114:131-38. doi: 10.1016/j. critrevonc.2017.04.007.

19. Cox CL, Montgomery M, Oeffinger KC, et al. Promoting physical activity in childhood cancer survivors: Results from the Childhood Cancer Survivor Study. Cancer 2009:115(3):642-54. doi: 10.1002/cncr.24043.

20. Mizrahi D, Wakefield CE, Fardell JE, et al. Distance-delivered physical activity interventions for childhood cancer survivors: A systematic review and meta-analysis. Crit Rev Oncol Hematol 2017;118:27-41. doi: 10.1016/j. critrevonc.2017.08.008.

21. Belle F, Wengenroth $L$, Weiss $A$, et al. Low adherence to dietary recommendations in adult childhood cancer survivors. Clin Nutr 2017:36(5):1266-74. doi: 10.1016/j. clnu.2016.08.012. Epub 2016 Oct 20.

22. Cohen J, Wakefield CE, Fleming CA, Gawthorne R, Tapsell LC, Cohn RJ. Dietary intake after treatment in child cancer survivors. Pediatr Blood Cancer 2012;58(5):752-57. doi: 10.1002/pbc.23280.

23. Russo S, Wakefield CE, Fardell JE, Cohn RJ. Summer sun-exposure in Australian childhood cancer survivors and community reference groups. Semin Oncol 2020;47(1):48-55. doi: 10.1053/j. seminoncol.2020.02.004.

24. Parsons HM, Jewett PI, Sadak K, Turcotte LM, Vogel RI, Blaes AH. e-Cigarette use among young adult cancer survivors relative to the US population. JAMA Oncol 2020;6(6):923-26. doi: 10.1001/jamaoncol.2020.0384.

25. Schulte F, Forbes C, Wurz A, et al. Childhood cancer survivorship: Daily challenges. Pediatr Clin North Am 2020;67(6):1083-101. doi: 10.1016/j. pcl.2020.07.004

26. Jefford M, Koczwara B, Emery J, Thornton-Benko E, Vardy JL. The important role of general practice in the care of cancer survivors. Aust J Gen Pract 2020;49(5):288-92. doi: 10.31128/AJGP-10-19-5133.
27. Vardy JL, Chan RJ, Koczwara B, et al. Clinical Oncology Society of Australia position statement on cancer survivorship care. Aust J Gen Pract 2019;48(12):833-36. doi: 10.31128/AJGP-07-194999.

28. Wakefield CE, McLoone J, Butow P, Lenthen K, Cohn RJ. Support after the completion of cancer treatment: Perspectives of Australian adolescents and their families. Eur J Cancer Care (Engl) 2013;22(4):530-39. doi: 10.1111/ecc.12059.

29. Donovan LA, Wakefield CE, Russell V, Cohn RJ. Hospital-based bereavement services following the death of a child: A mixed study review. Palliat Med 2015;29(3):193-210. doi: 10.1177/0269216314556851. 